\title{
PRIMEVAL STARBURST AND BULGE FORMATION
}

\author{
Keiichi WADA and Asao HABE \\ Department of Physics, Hokkaido University, Sappro 060 Japan
}

\section{Introduction}

Starburst phenomena in galaxies should have been common in the epoch of galaxy formation. How did the primeval starbursts affect the structure or the properties of the host galaxies?

Recently, some high-redshift radio-galaxies with a superwind and a secondly formed stellar system along radio lobe axis is observed(McCarthy et al. 1992). It is suggested that the expanding super bubble in a gaseous halo triggers the formation of the stellar system(Heckman et al. 1990).

Many hydrodynamical simulations of superwind originated in multiple supernovae showed that the superwind interacts with the ambient gas and forms a hot cavity and a cooled dense gas shell (e.g. Tomisaka and Ikeuchi 1988). Star formation in the gas shell should occur, if the gas shell radiatively cools and becomes gravitationally unstable(McCray and Kafatos 1987). We can interpret that part of the stellar systems observed in high-redshift galaxies were secondly formed during these process.

According to our estimate, the stellar shell secondly formed by super bubble associated with the primeval starbursts could have several $10^{10} M_{\odot}$ and several $k p c$ radius. We study the dynamical evolution and the effects of the relaxation of such stellar system on the structure of host galaxies. In the present paper we aim to clear basic physics of the evolution of the (shell + disk) stellar system by 3 -D N-body simulations.

\subsection{METHOD}

\section{Method and Models}

The N-body calculations were performed on the workstation with GRAPE-3. GRAPE(GRAvity PipE) is a series of special-purpose computers to simulate many-body systems, developed at University of Tokyo (Sugimoto et al. 1990). GRAPE calculates directly the gravitational force between particles, and the host computer performs all other calculations such as time integration.

\subsection{MODELS}

The stellar shells were calculated under two type of environments; (a)in a fixed external disky potential(Miyamoto and Nagai 1975): (b)in a thin stellar disk. The initial shells have uniform surface density; no rotation; and 
thin spherical shape. Parameters are mass of $\operatorname{shell}\left(M_{s}\right)$ and initial velocity dispersion of the shell $\operatorname{stars}\left(\sigma_{s}\right)$.

\section{Summary of our results}

1. The relaxation processes of the shells differ depending on $M_{s}$ and $\sigma_{s}$ and so do the finally formed bulge;

(a) Type $I$ : Relaxation by phase-mixing caused by the disk potential. A large and vague bulge is formed. The relaxation time is greater than $10^{9} \mathrm{yr}$.

(b) Type II : Relaxation dominated by self-gravity of the shell under the influence of the disk potential.

A compact core with a diffuse halo is formed in a following way $t<$ several $\left.10^{8} y r\right)$

i. Cylindrical/torus structure appears in the early stage of the relaxation.

ii. A few clumps are formed in this expanding torus at the disk plane by gravitational instability.

iii. They merge and form the bulge.

(c) Type III : Relaxation dominated by self-gravity. A triaxial bulge is formed.

2. The structure of the disk is changed during the relaxation of the shell. The disk thickens and non-axisymmetrical structure disappears due to the heating of the disk stars by the gravitational interaction between the disk and the shell stars.

3. The angular momentum of the disk is transferred to the shell stars during the relaxation.

\section{Acknowledgments}

We would like to thank Professors D.Sugimoto and T.Ebisuzaki, and Dr J.Makino for letting us use their GRAPE-3. We alse acknowledge the Nukazawa Memorial Foundation for our travel support.

\section{References}

Heckman,T.M. et al. 1990. Ap. J. Supplement 74, 833.

McCarthy, P.J., Persson, S.E., \& West, S.C. 1992. Astrophys. J., 336,52. McCray, R. \& Kafatos, M., 1987. Astrophys. J., 317, 190.

Sugimoto, D. et al. 1990. Nature, 345, 33.

Tomisaka, K. \& Ikeuchi, S. 1988. Astrophys. J., 330,695. 